\title{
Performance Analysis of Tunable Band Pass Filter and VCO for Multiband RF Front End
}

\author{
J.Manjula*, S.Malarvizhi \\ ECE department, SRM University, Kattangulathur, Tamil Nadu-603203, India \\ *Corresponding author's Email: jmanjulathiyagu@gmail.com
}

\begin{abstract}
This paper presents a design and performance analysis of tunable RF front end circuits such as RF band pass filter and VCO for multiband applications. The tunable element is an active inductor, built by MOS transistors. It is attractive due to its tunable and larger inductance values. The RF band pass filter is realized using active inductor with suitable input and output buffer stages. The tuning of center frequency for multiband operation is achieved through the controllable current source of the active inductor. The tunable range of the band pass filter varies from $3.9 \mathrm{GHz}$ to $12.3 \mathrm{GHz}$. The simulation results of band pass filter have minimum noise figure of $23 \mathrm{~dB}$ and has less power dissipation of $2.83 \mathrm{~mW}$. The simulated IIP3 is $-9.6 \mathrm{dBm}$ for 1 st and 3rd order frequency of $7.94 \mathrm{GHz}$ and 7.93 $\mathrm{GHz}$ respectively. The $\mathrm{VCO}$ designed using active inductor has the tuning range of $0.384 \mathrm{GHz}$ to $1.620 \mathrm{GHz}$ with the phase noise of $-139 \mathrm{dBc} / \mathrm{Hz}$ at the offset of $1 \mathrm{MHz}$. It consumes less power of $1.754 \mathrm{~mW}$ with the figure of merit of $189 \mathrm{dBc} / \mathrm{Hz}$. The designed active inductor, RF band pass filter and VCO are simulated in 180nm CMOS process using Synopsys simulation tool.
\end{abstract}

Keywords: Active inductor; Input impedance; Phase noise; Multi band RF front end

\section{Introduction}

The design of multi-band RF front end has stimulated the development of compact and hardware sharing transceivers in RF systems. CMOS technology plays a vital role in the design of highly integrated, low power and low cost RF systems. The block diagram of a multi-band RF front end is shown in Fig.1. An analog $\mathrm{RF}$ band pass filter is an essential block of RF fron$t$ end to select interested band in the received signal over entire spectrum. Low noise figure, low power consumption, tuning center frequencies and better linearity are some of the major challenges in the design of multiband RF band pass filter. Voltage controlled oscillator (VCO) is an important block of the RF front end which is used in frequency synthesizers to achieve absolute synchronization of local oscillator (LO) signals. The important specifications of VCO are center frequency, tuning range, power supply voltage, power consumption and phase noise. Most of the RF bandpass filters and VCO are implemented using on-chip spiral inductors [1]. But the spiral inductors cannot be realized for larger inductance values, high quality factor and smaller chip area [2]. On the other hand, active inductors can be realized for large inductance value with high resonance frequency, high quality factor, small chip area and wide range of tuning ability [3-6].

Active filters in $\mathrm{GHz}$ range have been designed in [7-8] and are not able to achieve the wide range of operating frequencies needed for multi standard RF systems. Widely tunable filters using varactors to change the operating frequencies have been designed in [9]. However, the varactors consume larger area and have less tuning range [10]. A fully integrated active LC band pass filter based on triple coupled spiral inductor topology has high power dissipation and less center 
frequency tuning range [11]. A second-order active band pass filter using integrated inductors implemented in Si bipolar technology consumes high power dissipation of $68 \mathrm{~mW}$ [12].

VCOs designed using active inductors are discussed in [13-16] have wide frequency tuning ranges and consume small amounts of power has shown an interest in the design of VCOs using active inductors. Furthermore, active inductor tuned VCOs are highly integrated and more cost effective than varactor tuned VCOs [17].

This task is to design a low power, low noise tunable RF band pass filter and VCO using active inductor for center frequency tuning. Section 2 briefly describes design and simulation results of active inductor, band pass filter and VCO.

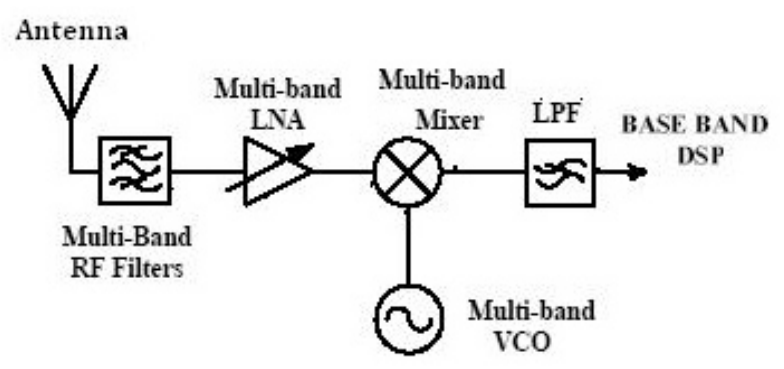

Figure 1 Block diagram of multi band RF front end

\section{Design and Simulation Results}

\subsection{Active inductor}

A single ended active inductor is realized using gyrator$\mathrm{C}$ topology [5] is an impedance inverter consisting of positive transconductor $G_{m 1}$ and negative transconductor $G_{m 2}$ connected back to back converts the port (parasitic) capacitances into an equivalent inductance. The proposed single ended active inductor with the aspect ratios in $\mu \mathrm{m}$ is shown in Fig. 2. It consists of differential pair M1 and M2 which represents the positive transconductor $G_{m 1}$ between the input (node 1) and the output (node 3). The cascode pair M3 and M4 represents the negative transconductor $-G_{m 2}$ between the input (node 3 ) and the output (node 1). Thus the $G_{m 1}$ and $-G_{m 2}$ forms the gyrator which converts the parasitic capacitance $C_{3}$ at node 3 to an equivalent inductance $L_{e q}=C_{3} / G_{m 1} G_{m 2}$. The passive equivalent circuit of the proposed single ended active inductor is shown in Fig.3.

The differential configuration of $G_{m 1}$ makes the proposed active inductor, less sensitive to noise and interference. The PMOS cascode structure of negative

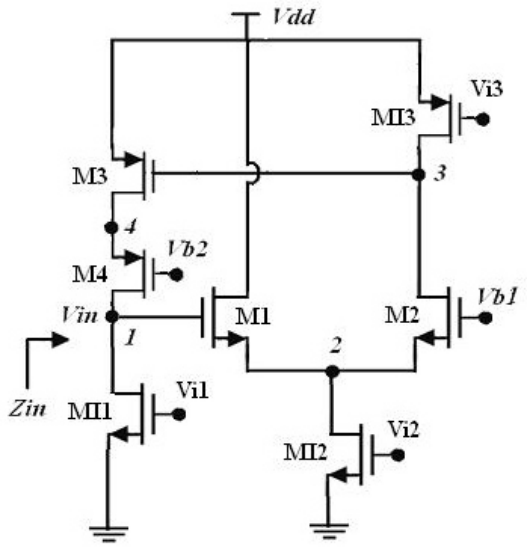

Figure 2 Circuit diagram of proposed single ended active inductor (with biasing arrangement) with the aspect ratios $(\mathrm{W} / \mathrm{L}$ in $\mu \mathrm{m})$ are $\mathrm{M} 1(2.25 / 0.18), \mathrm{M} 2(2.25 / 0.18)$, M3(4.5/0.18) \& M4(4.5/0.18).

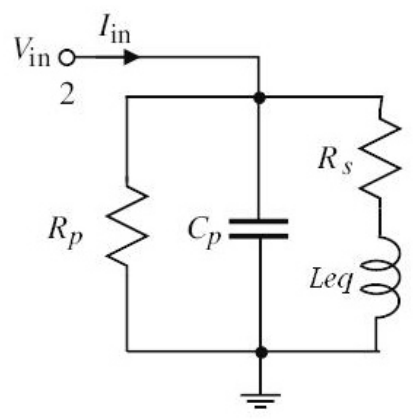

Figure 3 Passive equivalent circuit

transconductor $-G_{m 2}$, leads to possible negative resistance in series with the equivalent inductor to compensate the inductor loss. Thereby, it enhances the quality factor of the active inductor. Also, the cascode structure provides frequency range expansion by lowering the lower bound of the frequency range, thus increases the inductive bandwidth. The p-channel transistors are preferred for cascode structure as they have low noise and can be placed in separate n-wells, thus eliminating the non- linear body effect [4]. Thus, the combination of the differential configuration of $G_{m 1}$ and cascode configuration of $-G_{m 2}$ offers higher inductive bandwidth, higher resonance frequency and less noise.

The equivalent input impedance $Z_{\text {in }}$, can be obtained from the small signal equivalent circuit of the active inductor where $g_{1-4}$ are the transconductances of $M_{1-4}, C_{1-4}, g_{1-4}$ are the total parasitic capacitances and conductances at nodes 1-4 respectively. 


$$
\operatorname{Zin}(s)=\frac{\frac{s g_{4}}{C_{1}}+\frac{g_{3} g_{4}}{C_{1} C_{3}}}{s^{2}+s\left[\frac{g_{1}}{C_{1}}+\frac{g_{1} g_{3} C_{2}}{G C_{1} C_{3}}+\frac{g_{3}}{C_{3}}-\frac{\omega^{2} C_{2}}{G}\right] g_{4}+\frac{g m_{1} g m_{2} g m_{3} g m_{4}+g_{1} G g_{3}\left[g m_{4}+g_{4}\right]}{G C_{1} C_{3}}}
$$

Where $G=g m_{1}+g m_{2}+g_{2}$.

The format of $Z_{\text {in }}$ shows that it is equivalent to an RLC network, as shown in Fig. 3. Theesf term in the numerator indicates the equivalent inductance and the real term indicates series resistance.

From equation (1), $L_{e q}$ and $R_{s}$ can be written as,

$$
\begin{aligned}
& L e q=\frac{g_{4} G C_{3}}{g m_{1} g m_{2} g m_{3} g m_{4}+g_{1} G g_{3}\left[g m_{4}+g_{4}\right]} \\
& R s=\frac{G g_{3} g_{4}}{g m_{1} g m_{2} g m_{3} g m_{4}+g_{1} G g_{3}\left[g m_{4}+g_{4}\right]}
\end{aligned}
$$

The parallel capacitance $C_{p}=C_{1}$ and the parallel resistance $R_{p}=1 / g_{2}$.

The active inductor of Fig. 2 with the given aspect ratios is simulated in $180 \mathrm{~nm}$ CMOS process using Synopsys HSPICE simulator. The gate bias voltages are kept as $\mathrm{Vb} 1=0.2 \mathrm{~V}$ and $\mathrm{Vb} 2=0.25 \mathrm{~V}$. The controllable current sources are $I_{1}=90 \mu \mathrm{A}, I_{2}=80 \mu \mathrm{A}$ and $I_{3}=100 \mu \mathrm{A}$ The small signal parameters, $g m_{1}=523 \mu \mathrm{S}, g m_{2}=$ $724 \mu \mathrm{S}, g m_{3}=273 \mu \mathrm{S}, g m_{4}=873 \mathrm{mS}, g_{1}=91 \mu \mathrm{S}, g_{2}=$ $84 \mu \mathrm{S}, g_{3}=769 \mu \mathrm{S}, g_{4}=109 \mu \mathrm{S}, C_{1}=1.87 \mathrm{fF}, C_{2}=1.53 \mathrm{fF}$, $C_{3}=4.03 \mathrm{fF}, C_{4}=3.81 \mathrm{fF}$ and $G=1331 \mu \mathrm{S}$ are found from the operating points.
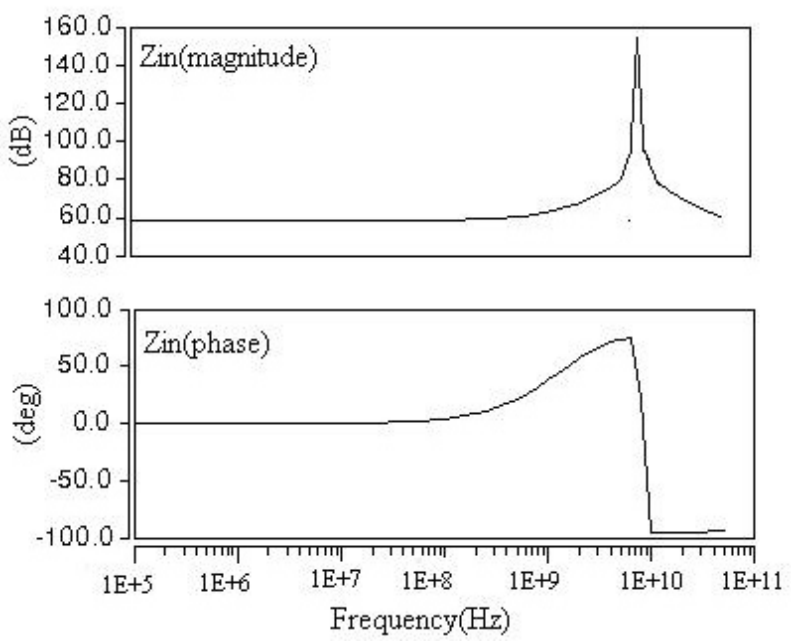

Figure 4 Simulated frequency response of input impedance

The simulated frequency response of $Z_{\text {in }}$ is shown in Fig. 4. The magnitude of $Z_{\text {in }}$ is nearly $150 \mathrm{~dB}$ and the phase change is from $+90^{\circ}$ to $-90^{\circ}$. The magnitude response shows that it has real term and imaginary ter$\mathrm{m}$. It is constant at $58 \mathrm{~dB}$ up to $1.5 \mathrm{MHz}$ which is equivalent to the real term. The real term is the series resistance $R_{s}$, which is calculated to be $154 \Omega$.

The response is increased from $1.5 \mathrm{MHz}$ to 7.94 $\mathrm{GHz}$ which is equivalent to the imaginary term, the equivalent inductance $L_{e q}$. The value of inductance ranges from $125 \mathrm{nH}$ to $1530 \mathrm{nH}$. Since it has less series resistance, the inductor loss is reduced. Fig. 5 shows the simulated inductance of the inductor.

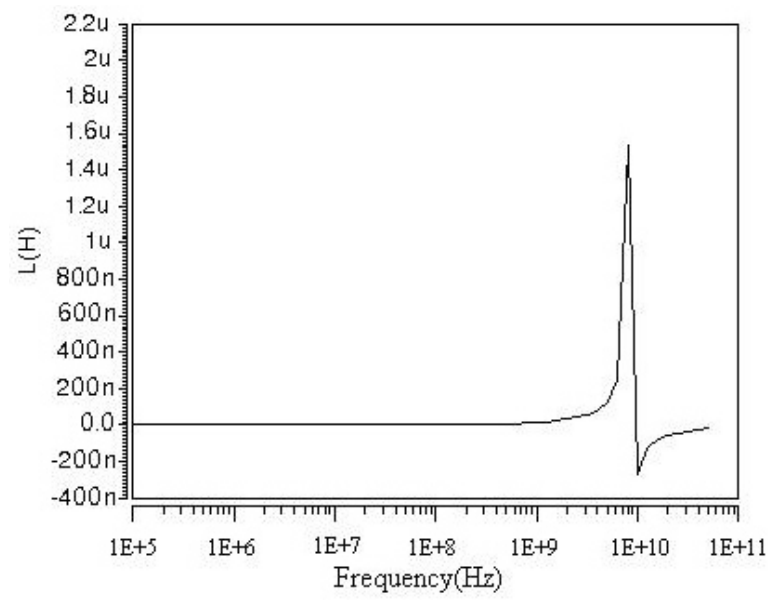

Figure 5 Simulated inductance

The quality factor $Q_{o}$ at $\omega_{0}$ is given as

$$
Q_{0}=\frac{\sqrt{\frac{g m_{1} g m_{2} g m_{4}+g_{1} G g_{3}\left[g m_{4}+g_{4}\right]}{G C_{1} C_{3}}}}{\left[\frac{g_{1}}{C_{1}}+\frac{g_{1} g_{3} C_{2}}{G C_{1} C_{3}}+\frac{g_{3}}{C_{3}}-\frac{\omega^{2} C_{2}}{G}\right] g_{4}}
$$

From the real and imaginary values of the simulation results, the quality factor $Q_{o}$ is calculated to be 497 at the frequency of $f_{o}=7.94 \mathrm{GHz}$. Fig. 6 shows the variation of $Z_{\text {in }}$ for different values of controllable current source $I_{2}$. When $I_{2}$ is varied from $50 \mu \mathrm{A}$ to $120 \mu \mathrm{A}$, the $Z_{i n}$ brings corresponding changes in $R_{S}$ and $L_{e q}$. Therefore, the quality factor is tuned through the controllable current source $I_{2}$. The resonance frequency $\omega_{0}$ is given as

$$
\omega_{0}=\sqrt{\frac{g m_{1} g m_{2} g m_{4}+g_{1} G g_{3}\left[g m_{4}+g_{4}\right]}{G C_{1} C_{3}}}
$$

The center frequency $f_{o}$ is tuned through the current source I3 of Fig. 2. Fig. 7 shows the tuning of 
the active inductor for various centre frequencies. The controllable current source $I_{3}$ is varied from $30 \mu \mathrm{A}$ to $100 \mu \mathrm{A}$ for tuning the centre frequency of the active inductor. The designed active inductor has wide tuning range of $3.9 \mathrm{GHz}$ to $12.3 \mathrm{GHz}$. Table 1 shows the tuning of active inductor for various center frequencies for different values of controllable current source $I_{3}$.

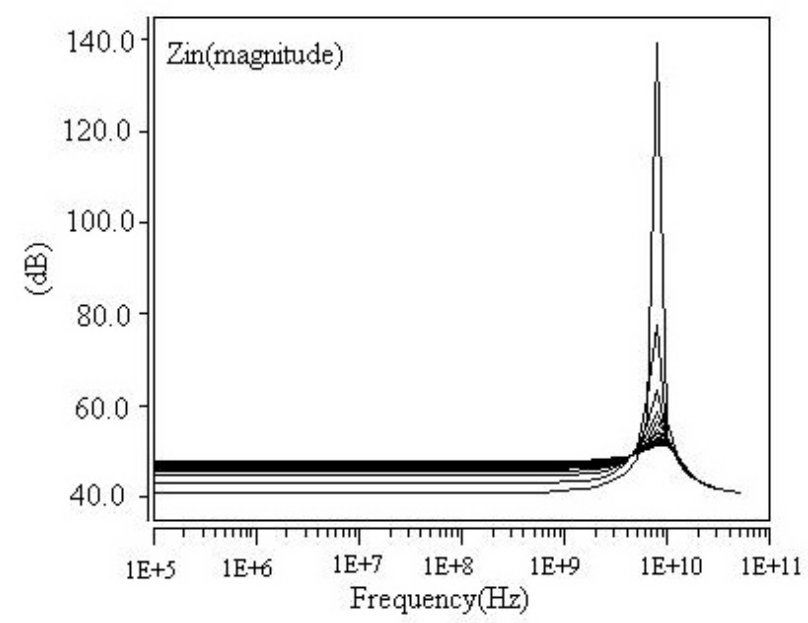

Figure 6 Quality factor tuning of the active inductor

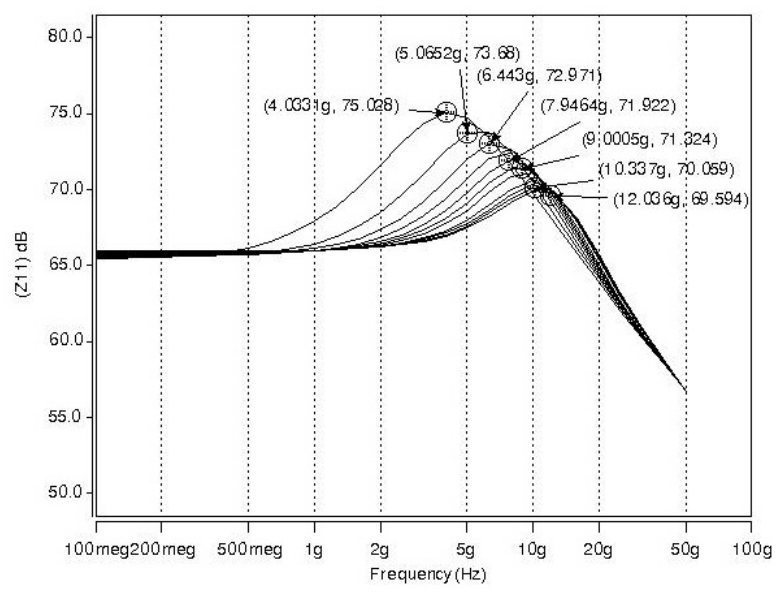

Figure 7 Center frequency tuning of the active inductor

The designed active inductor also features low power dissipation of $0.6 \mathrm{~mW}$. The noise output voltage varies from of $21 \mathrm{nV} / \sqrt{\mathrm{Hz}}$ to $7 \mathrm{nV} / \sqrt{\mathrm{Hz}}$ for the tuning range $3.9 \mathrm{GHz}$ to $12.3 \mathrm{GHz}$. Fig. 8 shows the noise voltage $\left(V^{2} / \mathrm{Hz}\right)$ as a function of frequency. Fig. 9 shows the simulated noise figure of $3.5 \mathrm{~dB}$ for the entire tuning range $3.9 \mathrm{GHz}$ to $12.3 \mathrm{GHz}$. Fig. 10 shows IIP3 of $-7.39 \mathrm{dBm}$ or $0.126 \mathrm{Vpp}$, which has been simulated for 1 st and 3rd order frequency of $7.94 \mathrm{GHz}$ and
Table 1 Center frequency tuning of active inductor

\begin{tabular}{l|l}
\hline $\begin{array}{l}\text { Controllable } \\
\text { current spurce }\end{array}$ & $\begin{array}{l}\text { Center } \\
\text { frequency }\end{array}$ \\
$I_{3}(\mu \mathrm{A})$ & $F_{0}(\mathrm{GHz})$ \\
\hline 72 & 3.99 \\
67 & 4.99 \\
64 & 6.3 \\
61 & 7.94 \\
55 & 9.9 \\
42 & 12.3 \\
\hline
\end{tabular}

7.93GHz respectively and has higher spurious free dynamic range of $118.7 \mathrm{~dB}$. The layout of the active inductor is shown in Fig. 11.

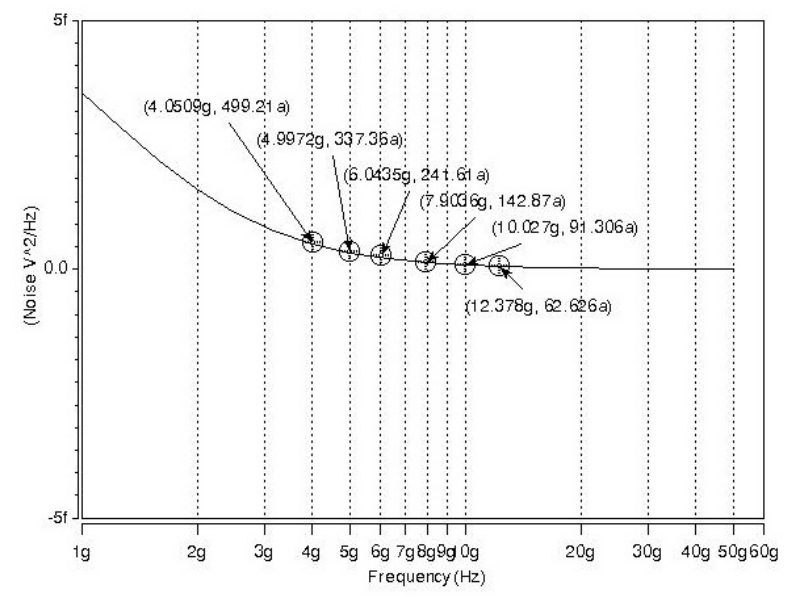

Figure 8 Noise voltage of the active inductor

\subsection{Multi band RF band pass filter}

The second order RF band pass filter of Fig. 12 is based on the active inductor topology. It consists of two resonators (M1, M2, M3, M4 and M5, M6, M7, M8) which are made up of single ended active inductors, coupled through the capacitance C [6]. Most of the band pass filter topologies used in thin film technology is of the coupled resonator type [18]. The advantage of coupled resonator filters is that they do not require a wide range of inductance values and are often realized using the same inductance for all resonators. The top coupled topology is one of the most commonly used. It is especially suitable to attenuate strong blocking signals in the cellular communications bands. However, in this type of filter the resonators operate in single ended mode. Min (W/L $=1 / 0.18$ in $\mu \mathrm{m})$ is the common gate transistor, is used as the input buffer stage for input matching. A source follower stage Mout (W/L=1/0.18 in $\mu \mathrm{m})$, is used as 


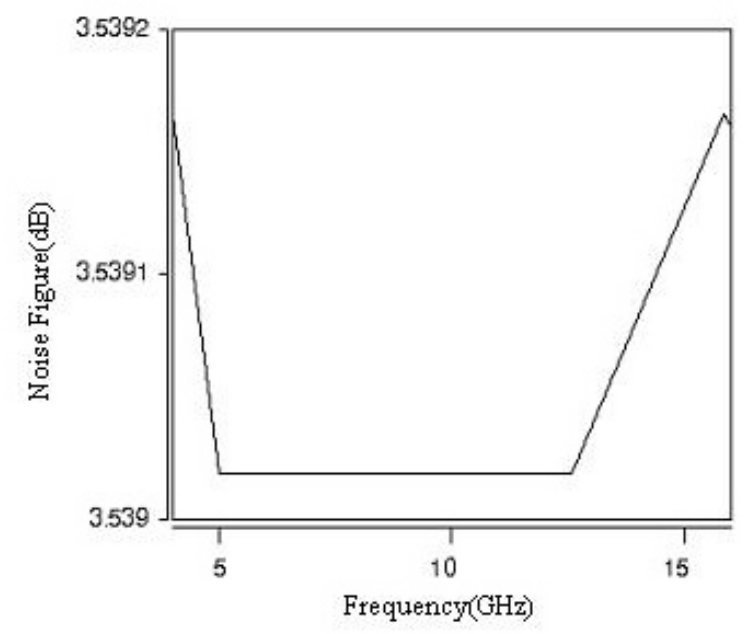

Figure 9 Noise figure of the active inductor

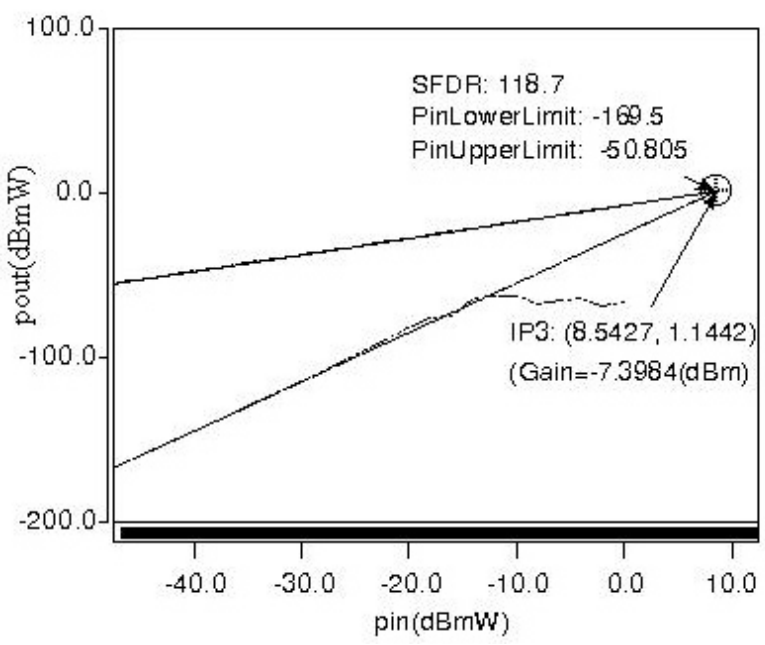

Figure 10 IIP3 of the active inductor

an output buffer stage for output matching and to reduce the loading effect. Rin and Rout are the input and output resistances which are selected to be $1 \mathrm{k} \Omega$. The controllable current source I6 is used for tuning the center frequency of the band pass filter.

The frequency response of the band pass filter with $1 \mathrm{~K} \Omega$ at both ends (Rin at source and Rout at load) is shown in Fig. 13. It is simulated for center frequency of $7.94 \mathrm{GHz}$ with gain of $28 \mathrm{~dB}$ and narrow bandwidth of $200 \mathrm{MHz}$ with less power consumption of $2.83 \mathrm{~mW}$.

With fixed $\mathrm{Vb} 1$ and $\mathrm{Vb} 2$, the center frequency can be tuned by varying controllable current source $I_{6}$ from $5 \mu \mathrm{A}$ to $110 \mu \mathrm{A}$. The tuning range of the band pass filter is $3.99 \mathrm{GHz}$ to $12.3 \mathrm{GHz}$. The tuning can be done through the single current source which adds advantage to this band pass filter. Table 2 lists the tuning of band pass filter for various center frequencies. Fig. 14 shows the simulation result of tuning of band pass filter for various center frequencies.

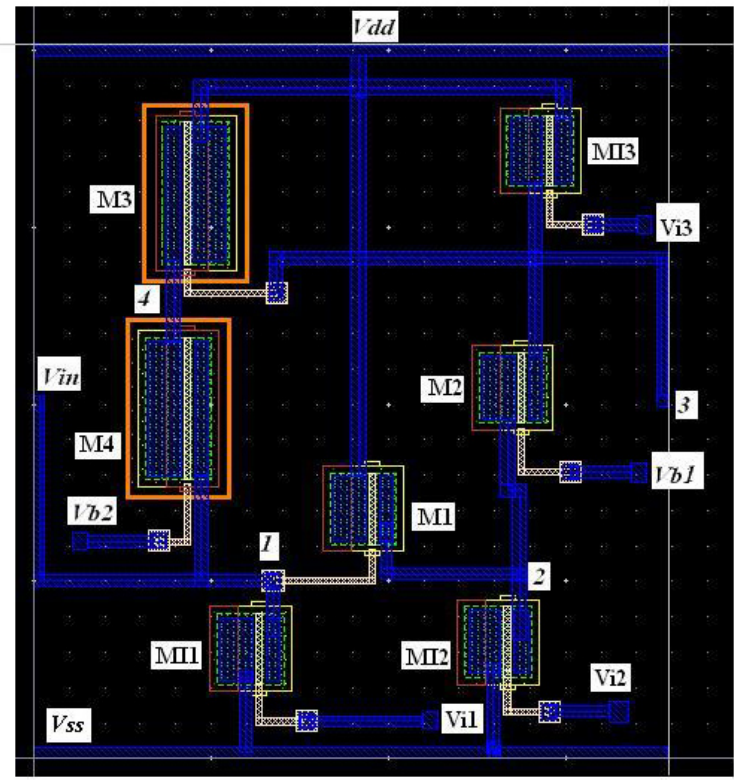

Figure 11 Layout of the active inductor

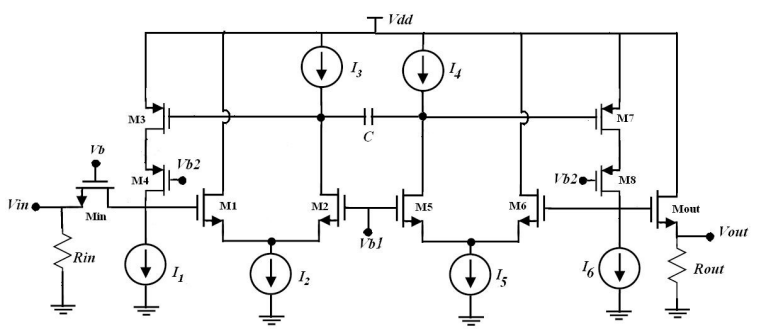

Figure 12 Circuit diagram of the RF band pass filter

The simulated noise figure ranges from $27 \mathrm{~dB}$ to $23 \mathrm{~d}$ B for the entire tuning range $3.9 \mathrm{GHz}$ to $12.3 \mathrm{GHz}$ as shown in Fig. 15. The noise figure is $23 \mathrm{~dB}$ for 7.94GHz. The IIP3 is $-9.6012 \mathrm{dBm}$ or $0.1 \mathrm{Vpp}$ which has been simulated for 1st and 3rd order frequency of $7.94 \mathrm{GHz}$ and $7.93 \mathrm{GHz}$ respectively as in Fig. 16. The $P_{1 d B}$ compression point is $-9.6 \mathrm{dBm}$ or $0.1 \mathrm{Vpp}$ from $50 \Omega$ source and higher spurious free dynamic range 94.101(dB) has been obtained.

\subsection{Tunable VCO}

The design of VCO is shown in Fig. 17. The topology of the VCO consists of cross coupled PMOS transistors to generate negative resistance and the resonant tank circuit [19]. The cross coupled PMOS transistors have smaller $1 /$ f noise due to lower mobility comparing to NMOS transistors, and they have less hot carrier effect [20]. Thus PMOS VCO can achieve better phase noise performance and suppression of power supply noise than NMOS VCO. The resonant tank 


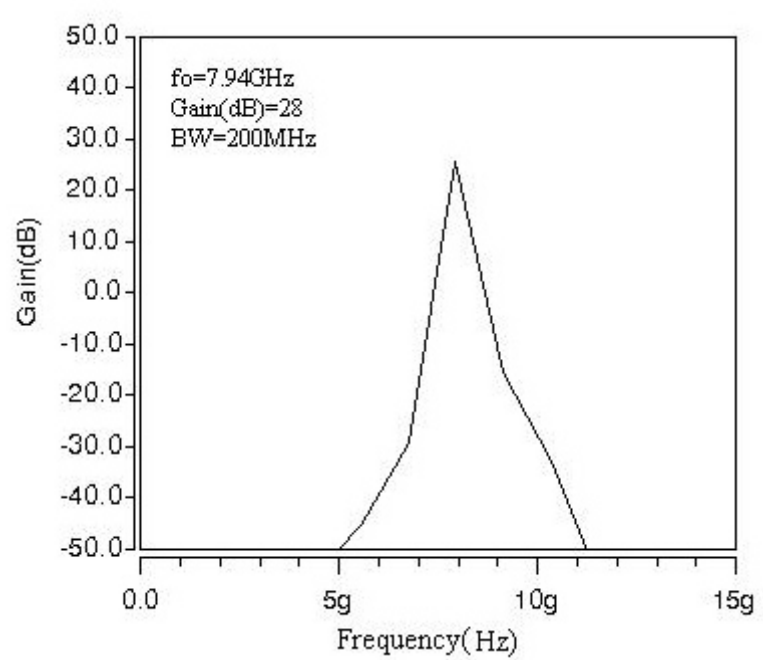

Figure 13 Frequency response of the band pass filter

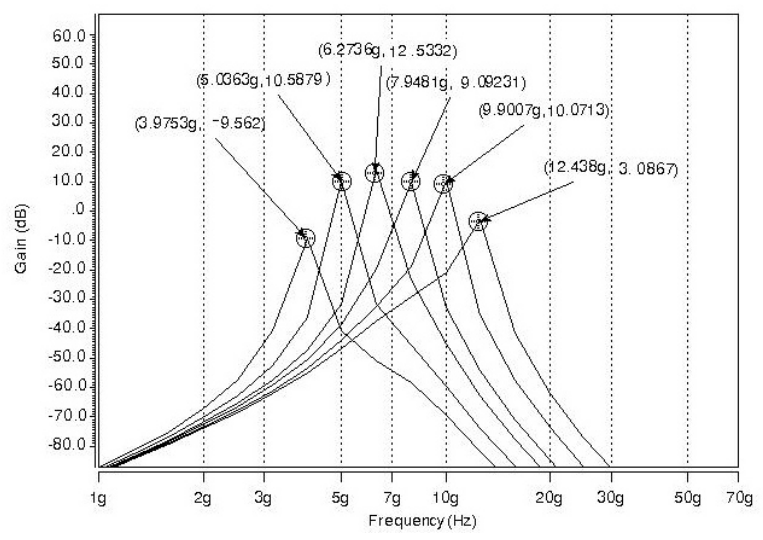

Figure 14 Tuning of band pass filter for various center frequencies

Table 2 Center frequency tuning of band pass filer

\begin{tabular}{l|l}
\hline $\begin{array}{l}\text { Controllable } \\
\text { current spurce }\end{array}$ & $\begin{array}{l}\text { Center } \\
\text { frequency } \\
I_{6}(\mu \mathrm{A})\end{array}$ \\
\hline 5 & $F_{0}(\mathrm{GHz})$ \\
10 & 3.99 \\
19 & 4.99 \\
35 & 6.3 \\
60 & 7.94 \\
110 & 9.9 \\
\hline
\end{tabular}

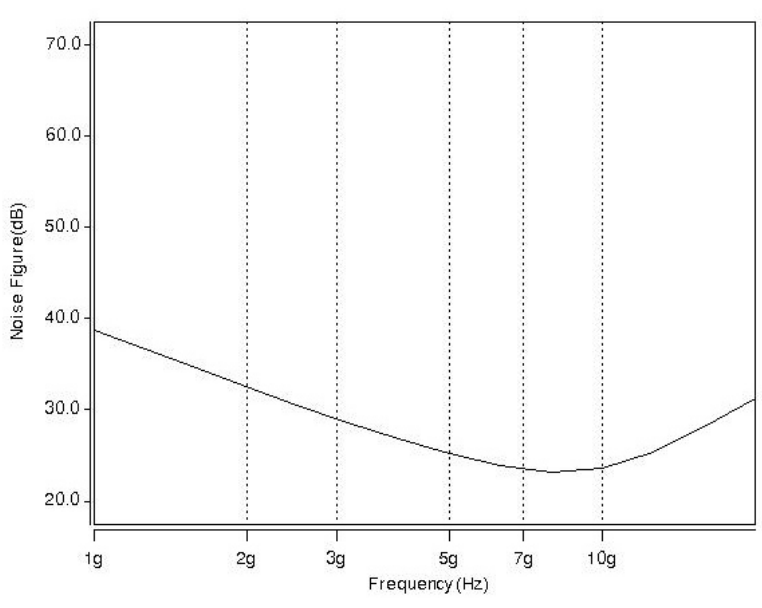

Figure 15 Noise figure of band pass filter

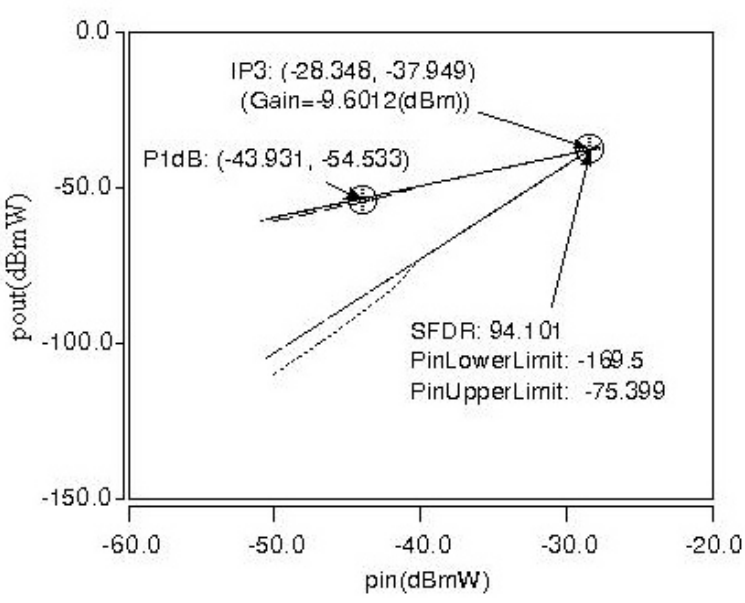

Figure 16 IIP3 of band pass filter

circuit is composed of a capacitor $\mathrm{C}$ and the active inductors L1 and L2. Active inductors are tunable and consume less power than spiral inductors. M3, M4, M5 and M6 forms the current reuse resistive feedback variable gain stage provides better output impedance matching. The oscillation frequency is given as equation (6) where $L_{e q 1}$ and $L_{e q 2}$ are the equivalent inductance of the active inductors L1 and L2 respectively. The equivalent inductances can be tuned through the controllable current source I 2 of the active inductors which in turn vary the oscillation frequency.

Fig. 18 shows Vout+ and Vout- of VCO at $f_{\text {osc }}=1.62 \mathrm{GHz}$ for $I_{2}=50 \mu \mathrm{A}$. Fig. 19 shows the VCO output waveforms for the different frequencies with respect to the current source $I_{2}$ of the active inductors. The tuning range of the $\mathrm{VCO}$ is $0.384 \mathrm{GHz}$ to 1.62GHz. Fig. 20 shows the simulated phase noise of $-139 \mathrm{dBc} / \mathrm{Hz}$ at the offset of $1 \mathrm{MHz}$. It consumes less power of $1.754 \mathrm{~mW}$ as shown in Fig 21, with the figure of merit of $189 \mathrm{dBc} \sqrt{\mathrm{Hz}}$. 


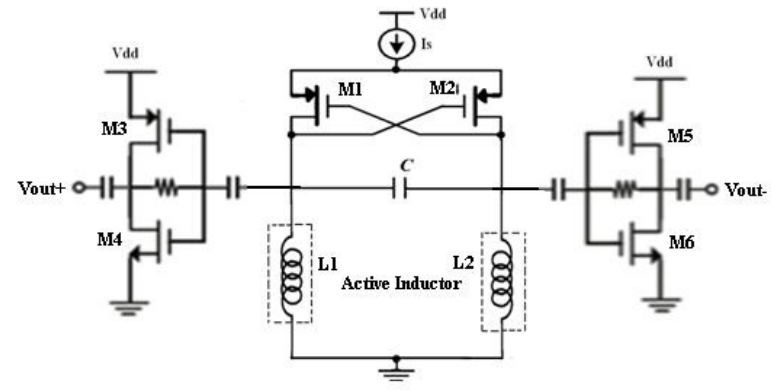

Figure 17 Circuit diagram of VCO

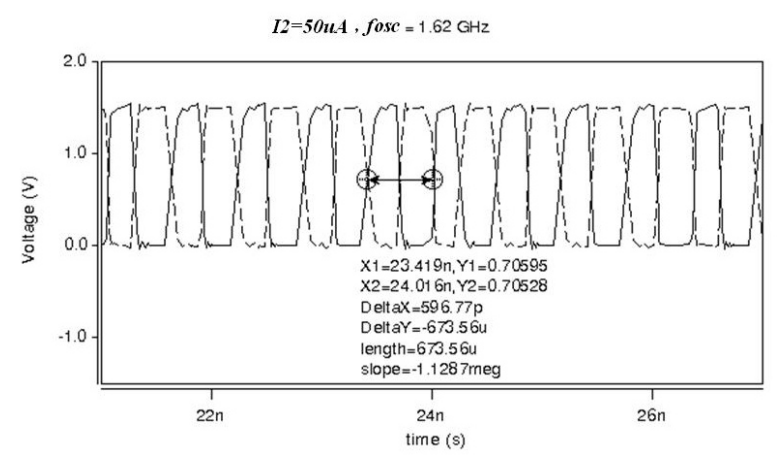

Figure $18 \mathrm{VCO}$ output waveform for $I_{2}=50 \mu \mathrm{A}$

\section{Performance Comparison}

Table 3 compares the performances of band pass filter with reported works in the literatures $[21,22]$ of R$\mathrm{F}$ band pass filters. The comparison results show that the band pass filter features wide range of center frequency tuning capability, less power dissipation and better linearity. Table 4 compares the performances of the VCO with the reported works in the literatures $[23,24,25]$ of VCOs. The comparison results show that the designed VCO features better tuning range and low power consumption.

Table 3 Comparison of band pass filter performances

\begin{tabular}{l|l|l|l}
\hline \hline Parameter & Ref[21] & Ref[22] & This Work \\
\hline Technology & $90 \mathrm{~nm}$ & $0.25 \mu \mathrm{m}$ & $0.18 \mu \mathrm{m}$ \\
& $1.2 \mathrm{~V}$ & $1.8 \mathrm{~V}$ & $1.8 \mathrm{~V}$ \\
\hline Filter order & 2 & 2 & 2 \\
\hline$\omega_{0}(\mathrm{GHz})$ & 3.46 & $1.6-2.45$ & $3.99-12.3$ \\
\hline $\begin{array}{l}\text { Power } \\
\text { dissipation } \\
(\mathrm{mW})\end{array}$ & 1.4 & 8.6 & 2.83 \\
\hline $\begin{array}{l}\text { NoiseFigure } \\
(\mathrm{dB})\end{array}$ & $5(\mathrm{at}$ & $17(\mathrm{at}$ & $23(\mathrm{at}$ \\
\hline IIP3 $(\mathrm{dBm})$ & -10.29 & -2.1 & -9.6 \\
\hline \hline
\end{tabular}
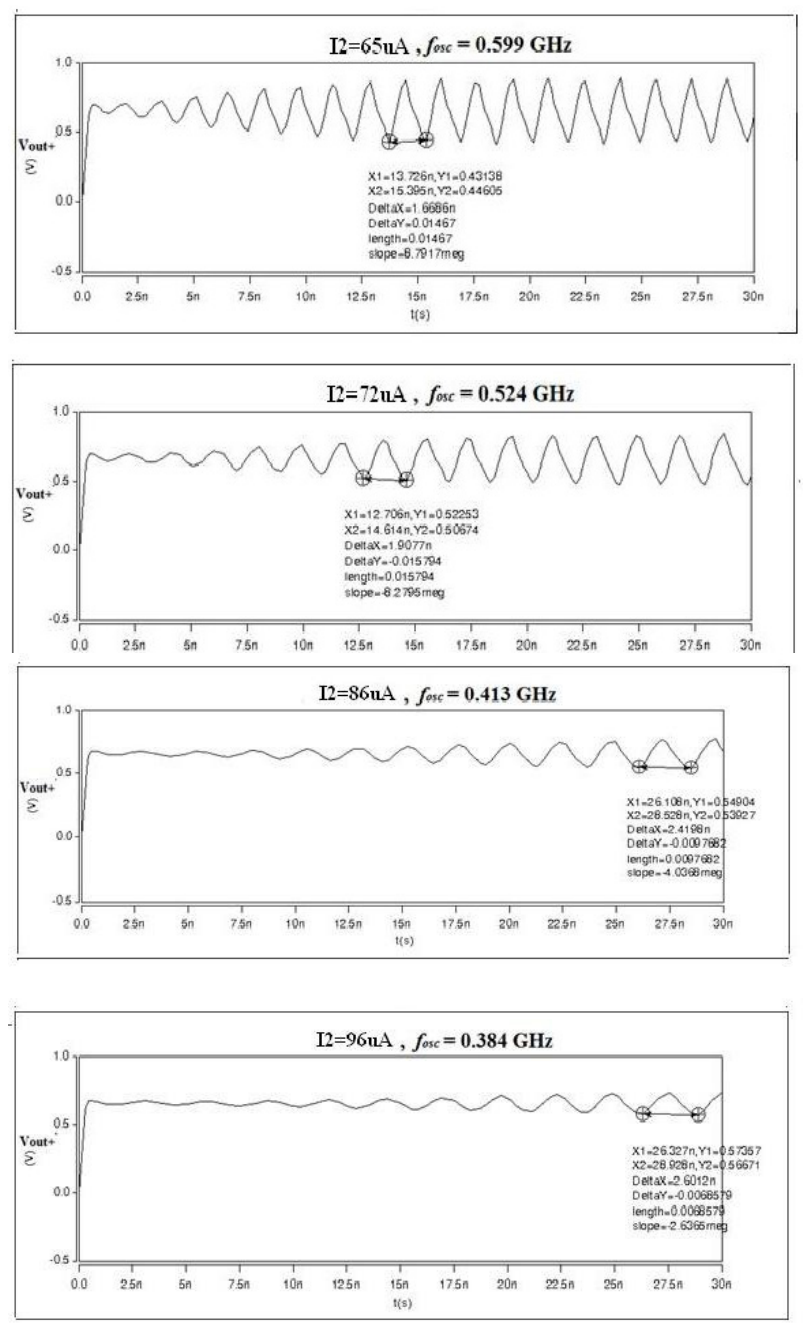

Figure $19 \mathrm{VCO}$ output waveforms for different values of $I_{2}$.

\section{Conclusions}

The tunable RF band pass filter and VCO based on CMOS active inductor are simulated in 180nm CMOS process. The simulation results of active inductor show that the circuit has wide inductive bandwidth and high resonance frequencies. The simulation results of RF band pass filter prove that it has better tuning of center frequencies, less noise and lower power dissipation. The simulation result of VCO features lower power and better tuning range. The designed RF band pass filter and VCO are more suitable to design low power $\mathrm{RF}$ front end circuits.

\section{References}

[1] W. B. Kuh, F. W. Stephenson, and A. ElshabiniRiad,"A $200 \mathrm{MHz}$ CMOS Q-enhanced LC band pass filter", IEEE Journal of Solid-State Circuits, Vol.31, No.8, pp.1112-1122, August 1996. 


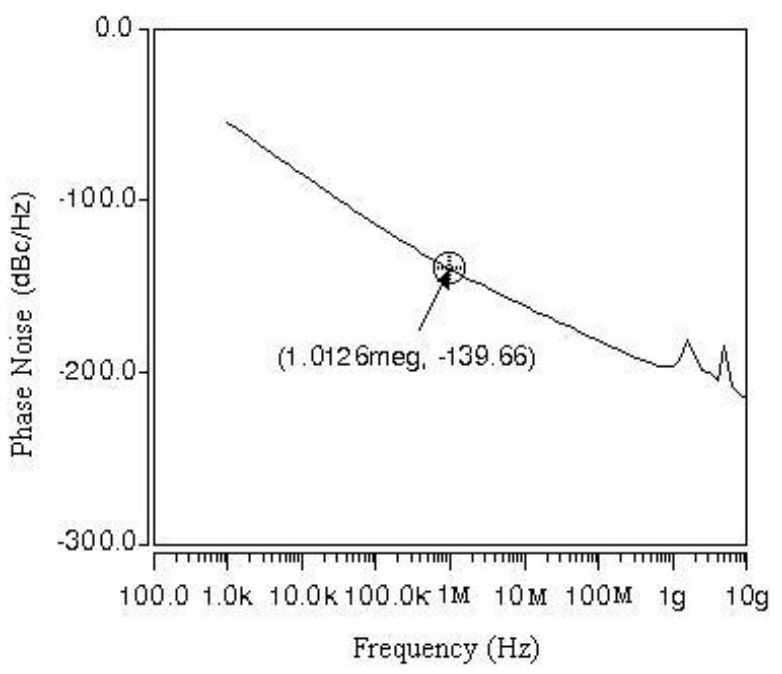

Figure 20 Simulated phase noise of VCO

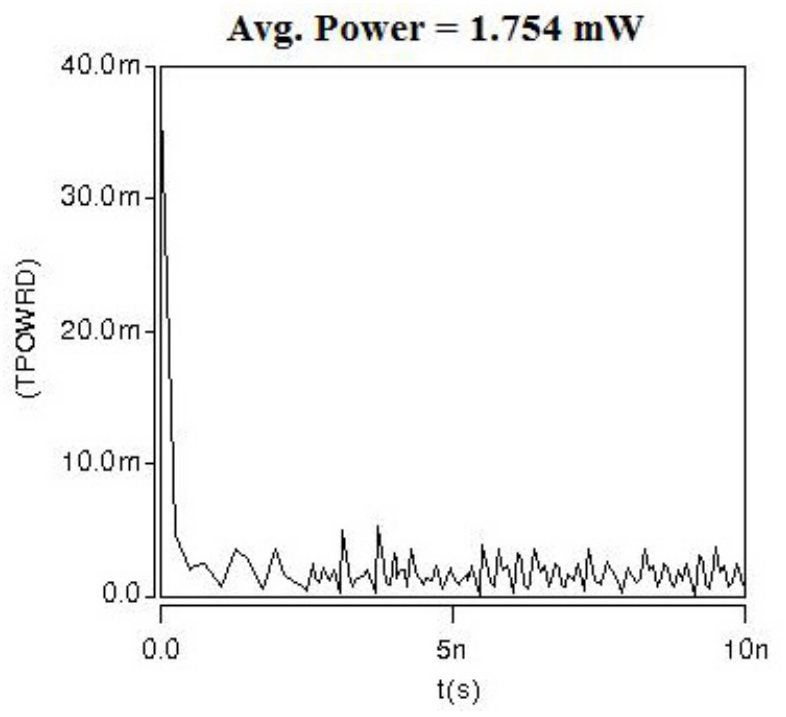

Figure 21 Average power consumption of VCO

[2] A. Thanachyanont,"Low-voltage low-power high-Q CMOS RF band pass filter”, Electronics Letters, Vol.38, No.13, pp.615-616, Jun 2002.

[3] H. Xiao and R. Schaumann, "A 5.4GHz high-Q tunable active-inductor band pass filter in standard digital CMOS technology", Analog Integrated Circuits and Signal Processing, Vol. 51, No. 1, pp. 1-9, 2007.

[4] A. Thanachayanont, "CMOS transistor-only active inductor for IF/RF applications", IEEE International Conference on Industry Technology (ICITfO2), Thailand, Vol.2, pp. 1209-1212, Dec 2002.

[5] A. Thanachayanont and A.Payne,"VHF CMOS integrated active inductor", Electronic Letters, Vol.32, No.11, pp.999-1000, May 1996.

[6] M.M Reja, I.M. Filanovsky and K.Moez,"Wide Tunable CMOS Active Inductor", Electronic Letters, Vol. 44, No.25, pp. 1461-1463, December 2008.
Table 4 Comparison of VCO performances

\begin{tabular}{l|l|l|l|l}
\hline \hline Parameter & Ref[23] & Ref[24] & Ref[25] & This Work \\
\hline $\begin{array}{l}\text { Supply } \\
\text { voltage(V) }\end{array}$ & 2.6 & 1.8 & $1.8 \mathrm{~V}$ & 1.8 \\
\hline $\begin{array}{l}\text { Technology } \\
(\mu \mathrm{m})\end{array}$ & 0.25 & 0.18 & 0.18 & 0.18 \\
\hline $\begin{array}{l}\text { Frequency } \\
(\mathrm{GHz})\end{array}$ & 1 & $2 / 5.8$ & 2.97 & 0.384 \\
\hline $\begin{array}{l}\text { Phase Noise } \\
(\mathrm{dBc} / \mathrm{Hz})\end{array}$ & -130 & $-112 /-107$ & -124.2 & -139 \\
at $1 \mathrm{MHz}$ & & & & \\
\hline $\begin{array}{l}\text { Power } \\
(\mathrm{mW})\end{array}$ & 9 & $11.7 / 9.3$ & 7 & 1.754 \\
\hline
\end{tabular}

[7] A. Thanachayanout and A.Payne,"CMOS floating Active Inductor and its Applications to Band Pass Filter and Oscillator Design", IEE Proceedings on Circuits, Devices and Systems, Vol. 147, No. 1, pp. 42-48, Feb 2000.

[8] F. Giannini, E.Limiti, G.Orengo and P.Sanzi,"High Q Gyrator based Monolithic Active Tunable Band stop Filter", IEE Proceedings on Circuits, Devices and Systems, Vol. 145, No. 4, pp. 243-246, August 1998.

[9] V. Aparin and P.Katzin,"Active GaAs MMIC Band Pass Filters with Automatic Tuning and Insertion Loss Control", IEEE Journal of Solid State Circuits, Vol. 30, No. 10, pp. 1068-1073, October 1995.

[10] W.M.Y. Wong, P.S.Hui, Z.Chen, K.Shen, J.Lau, P.C.H. Chan and P.Ko, "A Wide Tuning Range gated Varactor", IEEE Journal of Solid State Circuits, Vol. 35, No. 5, pp. 773-779, May 2000.

[11] S. Bantas, Y. Koutsoyannopoulos, "CMOS activeLC band pass filters with coupled inductor Qenhancement and center frequency tuning", IEEE Trans. Circuits and Systems-II: express briefs, Vol. 51, pp.69-77 Feb. 2004.

[12] S.Pipilos, Y.P. Tsividis, J. Fenk, and Y. Papanaos, “A Si $1.8 \mathrm{GHz}$ RLC filter with tunable center frequency and quality factor", IEEE J. Solid-State Circuits, Vol. 31, pp. 1517-1525, Oct. 1996.

[13] J. Laskar, R. Mukhopadhyay, and C.-H. Lee,"Active inductor-based oscillator:A promising candidate for low-cost low-power multi-standard signal generation", in Proc. IEEE Radio Wireless Symp., Jan. 2007, pp.31-34.

[14] L.-H. Lu, H.-H. Hsieh, and Y.-T. Liao,"A wide tuning-range CMOS VCO with a differential tunable active inductor", IEEE Trans. Microw. Theory Tech., vol. 54, no. 9, pp.3462-3468, Sep. 2006.

[15] R. Mukhopadhyay, Y. Park, P. Sen, N. Srirattana, J. Lee, C.-H. Lee, S. Nuttinck, A. Joseph, J. D. Cressler, and J. Laskar,"Reconfigurable RFICs in Si-based technologies for a compact intelligent RF 
front-end", IEEE Trans. Microw. Theory Tech., vol. 53, no. 1, pp.81-93, Jan. 2005.

[16] Y. Wu, M. Ismail, and H. Olsson,"CMOS VHF/RF CCO based on active inductors", Electron. Lett., vol. 37, no. 8, pp.472-473, Apr. 2001.

[17] Ulrich L. Rohde, Ajay K.Poddar,“Tunable Active Inductor Offers Integrable And Cost-Effective Alternatives of Varactor Tuned VCOs", IEEE International Frequency Control Symposium, pp. 962-967, April 2009.

[18] Robert C Frye, Kai Liu, Guruprasad Badakere, Yaojian Lin,“A Hybrid Coupled - Resonator Band pass Filter Topology Implemented on Lossy Semiconductor Substrates", IEEE/MTT-S International Microwave Symposium, pp. 1757-1760, June 2007.

[19] Shirin Bahramirad, Jad G. Atallah, Steffen Albrecht,"A Low Phase Noise VCO for Multi Band Wireless Transceivers", International Conference on Design \& Technology of Integrated Systems in Nanoscale era, pp. 148 -153, Sep. 2007.

[20] J. Y. Chen, "CMOS Devices and Technology for VLSI", Englewood Cliffs, NJ: Prentice Hall, Chapter 5, 1990.

[21] Santhosh Vema Krishnamurthy, Kamal El-Sankary, and Ezz El-Masry,"Noise-cancelling CMOS Active Inductor and Its Application in RF Band-Pass Filter Design", International Journal of Microwave Science and Technology, Vol. 2010, Article ID 980957, pp. 1 - 8, 2010.

[22] S. Bantas, Y. Koutsoyannopoulos, "CMOS activeLC band pass filters with coupled inductor Qenhancement and center frequency tuning", IEEE Trans. Circuits and Systems-II: express briefs, Vol. 51, pp.69-77, Feb. 2004.

[23] Z. Tang, J. He, H. Min, “A Low Phase Noise 1-GHz LC VCO Differentially Tuned by Switched Step Capacitors", IEEE Asian Solid State Circuits Conference, 2005.

[24] M. Yeh, W. Liou, T. H. Chen, Y. C. Lin, J. Ho,"A Low Power 2/5.8 GHz CMOS LC VCO for Multi band Wireless Communication Applications", International Conference on Communication, Circuits and Systems, 2006.

[25] B. Q. Diep, C. S. Park,“All PMOS Wideband VCO for Multi band Multi Standard Radios", The 9th International Conference on Advanced Communication Technology, 2007. 